\title{
Estrategias empresariales aplicadas en el proceso productivo de las industrias de pastas alimenticias del estado Zulia*
}

\author{
Meleán Romero, Rosana** \\ Nava Vásquez, Yuneska***
}

\section{Resumen}

En el presente artículo, se analizan las estrategias empresariales aplicadas por las industrias de pastas alimenticias del estado Zulia para la organización de su proceso productivo. La investigación es de tipo descriptiva, bajo un enfoque no experimental de campo; se inicia con una breve descripción del contexto alimenticio para posteriormente realizar un análisis teórico-práctico de las estrategias intra empresa aplicadas en los procesos productivos de la industria. La información empírica se recopiló a través de un cuestionario aplicado a los gerentes de plantas de las unidades de análisis. Los resultados indican que las grandes empresas han adoptado estrategias empresariales dirigidas hacia el interior de sus instalaciones; han modernizado sus procesos adoptando tecnología de producción automatizada para la fabricación de pasta larga y pasta corta, además de la estrategia de subcontratación para externalizar el proceso de elaboración de especialidades en empresas artesanales; por otra parte, en la empresa pequeña no se evidencia la aplicación de estrategias empresariales, la tecnología de producción empleada es mecanizada y no cuenta con especialidades dentro de su cartera de productos. Se concluye que en las grandes empresas la modernización de las líneas de producción de pasta larga y corta, ha permitido reorganizar procesos, mejorándolos continuamente, y a pesar que se han reducido puestos de trabajo, la cantidad es mínima para hablar de flexibilización numérica; las razones fundamentales para subcontratar la fabricación de especialidades es la reducción de costos, mientras que en la empresa pequeña no se presencian ninguna de estas estrategias.

Palabras clave: Estrategias empresariales, proceso productivo, modernización tecnológica, externalización, industrias de pastas alimenticias.

Recibido: 25-09-06. Aceptado: 18-07-07

* Este artículo es resultado del Programa de Investigación: Estrategias de Modernización Empresarial, Adscrito al Centro de Estudio de la Empresa (CEE), Facultad de Ciencias Económicas y Sociales (FCES), Universidad del Zulia.

** Investigadora del Centro de Estudios de la Empresa de la Facultad de Ciencias Económicas y Sociales (FCES). Universidad del Zulia. E-Mail: rosanamelean@cantv.net.

*** Investigadora del Centro de Estudios de la Empresa de la Facultad de Ciencias Económicas y Sociales (FCES). Universidad del Zulia. E-Mail: yuneskanava@cantv.net. 


\section{Business Strategies Applied to the Productive Process for the Pasta Industry in the State of Zulia}

\section{Abstract}

This article analyzes the business strategies applied by pasta industries in the State of Zulia for organizing their productive processes. The research is of a descriptive type, using a non-experimental, field approach; it begins with a brief description of the food industry context in order to later carry out a theoretical-practical analysis of the intra-company strategies applied in the industry's productive processes. Empirical information was collected using a questionnaire applied to the plant managers for the analysis units. Results indicated that large companies have adopted business strategies directed toward the interior of their installations; they have modernized their processes by adopting automated production technology for manufacturing long and short pasta, in addition to the strategy of subcontracting in order to externalize the process for making specialties in cottage industries. On the other hand, small companies do not apply business strategies; their production technology is mechanized and they do not include specialties in their product portfolio. Conclusions are that in large companies, the modernization of production lines for long and short pasta have allowed for reorganizing processes, continually improving them and despite reducing the number of jobs, the amount is minimal to talk about numerical flexibilizing. The fundamental reason for subcontracting specialty manufacture is cost reduction, while in the small companies, none of these strategies are present.

Key words: Business strategies, productive process, technological modernization, externalization, pasta industry.

\section{Introducción}

El sector alimenticio en el país está representado por un gran número de empresas; estas se clasifican en grandes, medianas o pequeñas pero independientemente de su tamaño están destinadas a la fabricación de productos dirigidos a satisfacer las necesidades alimenticias de la población.

Los alimentos, representan sin duda un insumo esencial para la sobrevivencia de los seres humanos; el arroz, los granos, las carnes, las legumbres, así como las pastas alimenticias constituyen un sector industrial importante para el fortalecimiento del aparato productivo nacional. En la medida que este sector crez- ca se generarán fuentes de empleo para la población, además de fomentar el desarrollo endógeno de nuestros pueblos.

Contradictorio a la tesis manejada por el gobierno nacional sobre el desarrollo desde adentro de las comunidades con potencial para ello, en Venezuela se siguen importando grandes cantidades de productos entre los que se destacan en los últimos tiempos los alimenticios; el pollo traído de Brasil y Argentina, los granos importados de Chile, el azúcar que se trae de Cuba, son puntos álgidos que llevan a la reflexión; la alimentación de los venezolanos no se debe dejar en manos de otros países. Hay que valorar el país que tenemos, rico en recursos naturales y humanos con calidad, que no son aprovechados. 
Debe ser tarea de los venezolanos crear sistemas de producción para la transformación de nuestra materia prima. En otras palabras, la tesis radica en poner en práctica los conocimientos en materia de producción e idear estrategias que permitan al emprendedor de hoy aprovechar los recursos naturales y convertirlos en productos con un mayor valor agregado.

El sector industrial encargado de la fabricación de pastas alimenticias, al igual que otros sectores de la economía venezolana, dada la no explotación del trigo con la calidad y cantidad exigidas para la producción de este tipo de productos, importa los insumos de países como Canadá y Estados Unidos; esto para iniciar la fabricación de las pastas en sus diferentes formatos y presentaciones.

La fabricación de estos productos alimenticios en las empresas que conforman el sector, poseen procesos productivos que a raíz de los acontecimientos económicos, políticos y sociales atravesados por nuestro país en los últimos años y los rápidos cambios y exigencias del mercado requieren de actualizaciones constantes, razón por la cual las empresas tratan de organizarlos adoptando estrategias para cubrir la cuota de mercado y obtener márgenes de rentabilidad aceptables.

En tal sentido, esta investigación pretende analizar las estrategias empresariales (intra empresa) asumidas por las industrias de pastas alimenticias del estado Zulia para la organización de sus procesos productivos. Para ello, se hace una breve descripción del sector; posteriormente, se realiza un análisis desde una perspectiva teórico-práctica de los procesos productivos de la industria y las estra- tegias empresariales aplicadas; la finalidad, es determinar que están haciendo las empresas para reorganizar sus procesos de trabajo, incrementar sus niveles de productividad y ocupar posiciones de prestigio en el mercado.

Para recopilar la información empírica, se entrevistó a personal clave en las organizaciones, aplicando un instrumento de recolección de información previamente construido y validado por reconocidos investigadores en el área.

\section{Estrategias empresariales en el sector alimenticio venezolano}

A comienzos del siglo $X X I$, en Venezuela se han generado importantes procesos de cambio, de los cuales no escapa el sector industrial; éste en la actualidad se encuentra inmerso en un contexto que impone retos económicos, laborales, políticos y gubernamentales, los cuales se deben afrontar para sobrevivir y/o conservar posiciones en el mercado.

Los procesos de cambio en el interior de las empresas van de la mano con las reglas impuestas por la globalización o mundialización, como hecho ineludible que trasciende las fronteras y obliga a las empresas a transformar el enfoque gerencial y comprometerse necesariamente a luchar por incrementar la productividad y crear ventajas competitivas sostenibles en el tiempo.

El reto globalizador y las constantes exigencias del entorno en cuanto a la modernización de los procesos internos de las empresas, y la capacidad para iniciar procesos de cambio; son puntos de partida para transformar la gestión empresarial, cambiar los paradigmas que 
han permanecido por años y buscar nuevas formas de gerenciar, es el reto que deben enfrentar las empresas venezolanas. En este sentido, Yip (1993: 1), establece que desarrollar y poner en práctica una estrategia global eficaz es la prueba de fuego de una compañía bien administrada.

Aunado a los proceso de cambio, en el ámbito empresarial, se configuran un $\sin$ fin de estrategias que responden tanto a necesidades internas como a aspectos del entorno. Dichas estrategias son susceptibles de analizar para adoptar aquellas que den respuestas a las exigencias tanto internas como externas de las empresas; sea la modernización tecnológica en producción, la reorganización de procesos (reingeniería o rediseño parcial), la flexibilización de las relaciones laborales, la diversificación o concentración de actividades, la externalización de procesos no medulares, entre otras. La adopción de estas estrategias conlleva al incremento de la productividad, la reducción de costos de producción y la creación de ventajas competitivas necesarias para alcanzar una posición de liderazgo en el mercado.

En esta investigación, puesto que el tema central es la organización de los procesos internos de las empresas (proceso de producción) se abordarán las estrategias dirigidas hacia el interior de las mismas (intra-empresa) (Gamboa y col. 2001 y 2003), específicamente las relacionadas con la modernización tecnológica en producción que conlleva a la adopción de estrategias de reorganización como el rediseño de procesos y a estrategias relacionadas con la fuerza laboral: flexibilización de las relaciones laborales. Además, dada las características de los procesos productivos de las empresas analizadas se considerará en el análisis la estrategia de subcontratación o externalización adoptada por las grandes empresas para el proceso de fabricación de especialidades.

Estas estrategias se identificarán y analizarán en un sector industrial constituido por tres empresas: Cargill de Venezuela, Molinos Sagra, C.A y La Comisana, las dos primeras calificadas como grandes y la tercera como pequeña, de acuerdo a lo establecido en el artículo 3 de la Ley para la promoción y el desarrollo de la pequeña y mediana industria ${ }^{1}$. Estas empresas proveen a la población zuliana las pastas en diferentes formatos y presentaciones, considerando la alta demanda existente en la región y en el país.

Según la Asociación Venezolana de Fabricantes de Pastas (2001), el consumo de pastas en Venezuela, es aproximadamente de 13 kilogramos por persona al año, uno de los más elevados del mundo, ocupando con esto el segundo lu-

1 Se considera pequeña industria; la planta de trabajadores con un promedio anual no menor de once (11) trabajadores, ni mayor a cincuenta (50); la Mediana Industria, mantiene un promedio anual no menor de cincuenta y un (51) trabajadores, ni mayor a cien (100) trabajadores. Atendiendo a esta clasificación, las empresas que cuentan con más de cien (100) trabajadores son consideradas grandes industrias. 
gar después de Italia; a finales de los años sesenta, era tan elevado el incremento de la demanda que pequeñas fábricas de carácter artesanal se vieron en la necesidad de incluir líneas automáticas de producción continua de 400 a $1000 \mathrm{~kg}$. por hora, que a finales de los 80 fueron reemplazadas gradualmente por modernas líneas de producción, que siguen actualizándose con el transcurrir de los años.

Por esta razón, la pasta en Venezuela constituye en la actualidad uno de los alimentos básicos del hogar venezolano, en los cuales se sirve como plato principal dos o tres veces por semana. Dada la alta demanda de pastas en el país, Venezuela ha buscado diferentes vías para incrementar la variedad de formatos y número de marcas, puesto que la novedad, moda y diversidad, atraen al consumidor venezolano y la competencia en el sector es fuerte.

El empresario venezolano, independientemente del sector en el que se desenvuelva, se encuentra en un constante dilema: adopta el cambio, actualizándose con los nuevos enfoques y corrientes empresariales o simplemente desaparece produciéndose la extinción de su empresa.

\section{Proceso productivo en las industrias de pastas alimenticias}

Las organizaciones, independientemente de la actividad económica a la que se dediquen, requieren de una serie de procesos para la concreción de sus productos finales, sean éstos intangibles en empresas de servicios o productos tangibles en empresas manufactureras.

La transformación de insumos en empresas manufactureras, se convierte en un elemento fundamental, la elaboración de cualquier tipo de productos requiere indiscutiblemente de una serie de actividades interrelacionadas que constituyen los procesos, considerados la médula espinal de cualquier organización dedicada a la fabricación o transformación de insumos en un bien con determinado valor social.

Clasificar los procesos depende del área funcional de la empresa en la cual se lleven a cabo, pueden existir procesos administrativos generales como los propuestos por Taylor y Fayol: planificar, organizar, dirigir y controlar las actividades de las empresas en cualquiera de sus áreas; o procesos aún más específicos u operativos, desarrollados en áreas empresariales específicas. Tal es el caso del proceso de fabricación o proceso de producción necesario para la elaboración de un producto determinado. Sin embargo, según Rodríguez y col. (2002) en las empresas que prestan servicios, se está utilizando el término servucción para hacer referencia al proceso llevado a cabo para la obtención de los mismos.

El proceso de producción, forma parte de la plataforma productiva de la empresa; además de ser el encargado de transformar insumos o materias primas en productos terminados o semi-elaborados destinados a la satisfacción de necesidades de la población; para ello requiere de medios de producción como por ejemplo instalaciones, equipos, tecnología, mano de obra o fuerza de trabajo y otros factores adicionales considerados necesarios para el logro de los objetivos planeados.

Un proceso de producción, también denominado proceso de fabricación, considerando una acepción simplista, se 
concibe como "cualquier actividad que produzca algo" (Sipper y Bulfin, 1998:7). Sin embargo, si se analiza a mayor profundidad, un proceso productivo engloba otros elementos.

Krajewski y Ritzman (2000: 3-4) lo definen como "cualquier actividad o grupo de actividades mediante los cuales uno 0 varios insumos son transformados y adquieren un valor agregado, obteniendo así un producto para el cliente". Por su parte, Rodríguez y col. (2002) comparten la definición planteada por Chase y col. (2005) quienes conciben un proceso productivo como aquel que utiliza recursos operacionales para transformar insumos en algún tipo de resultado deseado, agregando que este resultado podría ser un producto terminado.

Para Chase y col. (2005), un proceso productivo es sinónimo de sistema de producción o sistema productivo; sin embargo, si se considera la apreciación planteada por Boyer (1998) citado por Lucena (2003:71), son términos que se deben conceptualizar de manera diferente. Boyer define un sistema de producción como:

"la complementariedad y coherencia entre: los principios de gestión, la organización interna de la firma, su articulación con las empresas subcontratistas y con la competencia, y la relación salarial... y el sistema educativo sin olvidar la regulación macroeconómica".

Al considerar la definición de Boyer, se aprecia la amplitud de la misma al incluir principios de gestión y organización interna de la empresa; esto engloba todas sus áreas funcionales, además al considerar las relaciones que mantiene con otras instituciones, se evidencia la interrelación o enfoque de sistema abierto. Esto conlleva a pensar que un sistema productivo, puede estar representado por una empresa, constituida por áreas funcionales que trabajan como un todo interrelacionado y conforma un sistema de producción que genera bienes y servicios destinados a satisfacer necesidades de la sociedad.

En lo que respecta al proceso de producción, éste se limita simplemente a la transformación de determinadas entradas representadas por materias primas 0 insumos (en el caso de las empresas de transformación) y de personas (en empresas de servicios), para generar en el primer caso, productos o bienes tangibles y en el segundo, bienes intangibles o clientes con alguna necesidad satisfecha.

Por otra parte, Fernández y col. (2003), proponen una definición más precisa sobre el proceso productivo, concibiéndolo como el conjunto de actividades por medio de las cuales los factores productivos entre ellos tierra, capital, tecnología y fuerza de trabajo se transforman en productos, creando riqueza y añadiendo valor a los componentes o inputs adquiridos por la empresa. Los citados autores en una nueva edición (2006), añaden las tareas, flujos y almacenamiento como partícipes en cualquier proceso de producción. Sin embargo, 1) una tarea forma parte de una actividad $^{2}$; 2) los flujos constituyen la secuencia lógica de todo proceso, y 3) el almacenamiento según Tamayo (1991) es considerado como una actividad que no agrega valor al producto que se elabora.

Así pues, partiendo de las definiciones anteriores el proceso productivo se puede definir como un conjunto de actividades o tareas interrelacionadas entre sí encargadas de transformar determinadas entradas en salidas que pueden estar 
representadas por un bien que puede ser un producto terminado o semi-terminado que se convierte en un insumo para cualquier otro proceso; para lograr dichas transformaciones, se necesita de medios de producción, entre ellos el esfuerzo físi$\mathrm{CO}^{3}$ de las personas en algunos casos, las instalaciones, la tecnología y un elemento sumamente importante en la actualidad: el conocimiento.

En las industrias analizadas, los procesos productivos, se pueden dividir en dos líneas de producción: 1) línea de producción para pasta larga y 2) línea de producción para pasta corta; ambas líneas en las grandes empresas están muy automatizadas y presentan gran rigidez, mientras que en la empresa pequeña, la tecnología de producción que poseen es mecanizada.

Por otra parte, las grandes empresas dado que comercializan ciertas especialidades como los ñoquis de papa, raviolis, tortelloni, pasta al huevo, entre otros, que no pueden ser fabricadas en éstas líneas de producción por su completa automatización y rigidez, subcontratan empresas artesanales para la elaboración de las mismas, delegando esta actividad productiva que diversifica la variedad de productos ofrecidos por las empresas.

En tal sentido, se tiene en el sector industrial de pastas alimenticias, fundamentalmente dos tipos de proceso pro- ductivos: 1) para la elaboración de pasta larga y pasta corta, con ligeras diferencias entre ambos y, 2) para elaboración artesanal de especialidades. Es importante destacar que los procesos para la elaboración de pasta larga y pasta corta son automatizados en las grandes empresas y mecanizado en la pequeña ${ }^{4}$.

\subsection{Producción automatizada de pasta larga y pasta corta}

Los procesos productivos para la fabricación de las pastas (larga y corta) en las empresas: Cargill de Venezuela, S.A. y Molinos Sagra, C.A. (Mosaca) cuentan con un proceso previo de molienda del trigo (desarrollado en un departamento denominado molienda), del cual se obtiene la harina o sémola, principales insumo en la fabricación de pastas alimenticias; mientras que la empresa clasificada como pequeña, la materia prima principal está representada por la harina de trigo, esta se adquiere en el mercado nacional de harinas, específicamente en la empresa Molinos Nacionales, C.A. (Monaca).

El proceso de elaboración de las pastas alimenticias, se inicia cuando la sémola o la harina de trigo, dependiendo la calidad de la pasta que se quiera, es transportada desde los silos de almacenamiento (departamento de molienda), pasando por unos distribuidores hasta los

2 Una actividad según el diccionario de la Real Academia Española (2006) es un conjunto de operaciones o tareas propias de una persona o entidad, mientras que según el Diccionario el mundo (2006) una tarea se puede concebir como sinónimo de actividad.

3 El esfuerzo físico es necesario en trabajos rudimentarios o artesanales, dónde la actividad humana se convierte en el principal recurso para la producción. 
silos de almacenamiento del pastificio o silos de línea; desde donde son trasportadas mediante transferencias internas por un sistema neumático, desplazándose por un cernidor o tamiz de control a las líneas de producción de pastas.

Cuando la sémola o harina es almacenada en los silos de línea, se pasa a un proceso de dosificación de la materia prima; que permite asignar el porcentaje de agua, harinas o sémolas dependiendo de la marca de pasta a producir; posteriormente pasa a la etapa de mezclado y amasado; en la cual interviene un equipo con paletas rotando a alta velocidad y unas toberas de inyección de agua en forma de rocío, lo que da un área de contacto agua/harina bastante grande; el proceso de amasado tiene como propósito, a través de las máquinas amasadoras, eliminar el aire de la masa que formaría burbujas en la pasta ya seca; esto degradaría la calidad del producto.

Seguidamente, la masa es extrusionada o prensada por unos tornillos extrusores que la compactan y le dan una estructura más homogénea, para luego forzarla a pasar por un molde que le da la forma según la pasta que se quiera (pasta larga o corta); la pasta corta es cortada en esta fase antes de pasar al proceso de secado $^{5}$.
Debido a que la masa aún está húmeda, debe ser sometida al proceso de secado, el cual debe ser gradual puesto que la humedad va emigrando lentamente de la parte interna a la parte externa de la pasta, por lo cual se realiza en fases distintas para cada uno de los formatos de pasta: corto y/o largo.

Posterior a esto, ya cuando la pasta está completamente seca y cortada, se produce un almacenaje temporal de la misma, para luego ser pesada y proceder al empaquetado y sellado; luego las pasta es enfardada en bultos de acuerdo a la cantidad de paquetes requeridos; estos son colocados en estibas de madera, las cuales son almacenadas para su posterior comercialización en el mercado de alimentos.

Es importante destacar que el proceso de elaboración de pastas alimenticias (larga y corta), es similar en las empresas analizadas; por esta razón se decidió integrarlo en uno solo para las tres empresas; resaltando que siempre existen cambios relativos a las tecnologías de producción y equipos utilizados, y en los tiempos y fases de secado $^{6}$ (Ver Diagramas 1 y 2 ).

Es evidente, que las grandes empresas en aras de competir en un mercado con un numero reducido de competidores (representado por tres empresas en el estado Zulia), se esfuercen constan-

$4 \quad$ Industria de tradición familiar y muy conservadora en sus procesos de trabajo.

5 Esta fase del proceso presenta cierta flexibilidad, es decir es el único cambio que permite la línea, representado por el cambio de los moldes dependiendo del formato de pasta que se quiera.

6 Es notoria la tecnología utilizada por las grandes empresas comparadas con la empresa pequeña, quien se apoya en medios de producción convencionales y muchas veces rudimentarios por no contar con suficiente flujo de caja (efectivo) para realizar inversiones tecnológicas que le permitan incrementar los niveles de productividad y competir con los grandes. 


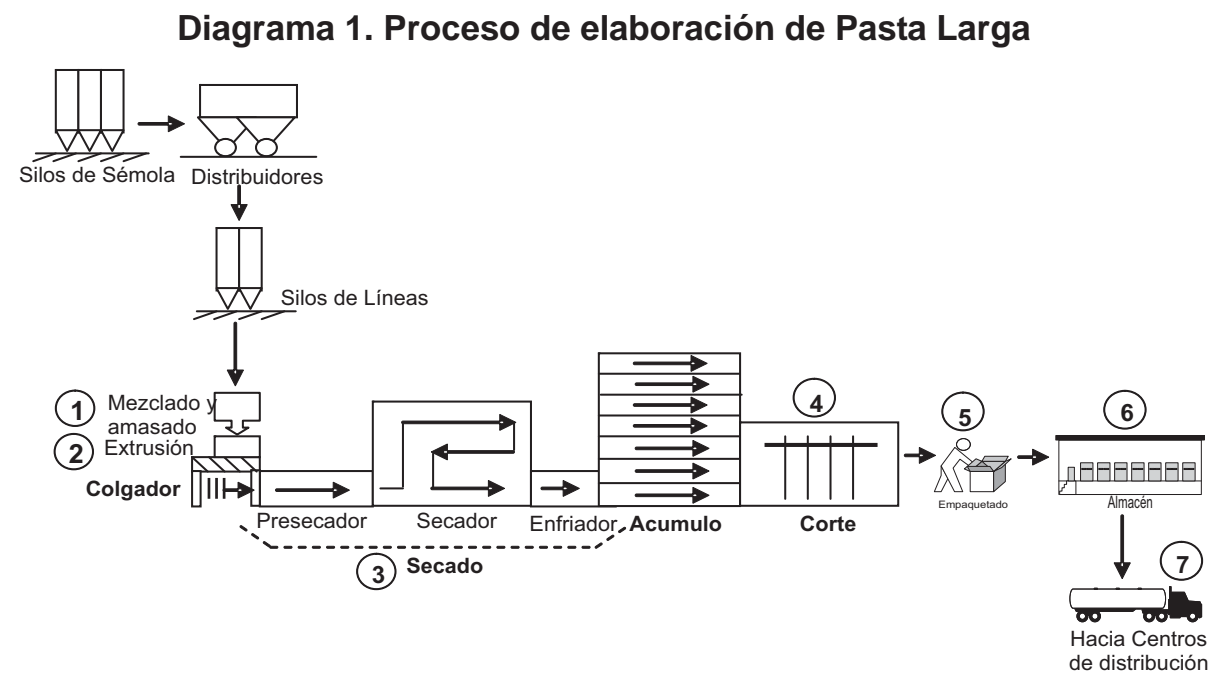

Fuente: Meleán (2004).

\section{Diagrama 2. Proceso de elaboración de Pasta Corta}

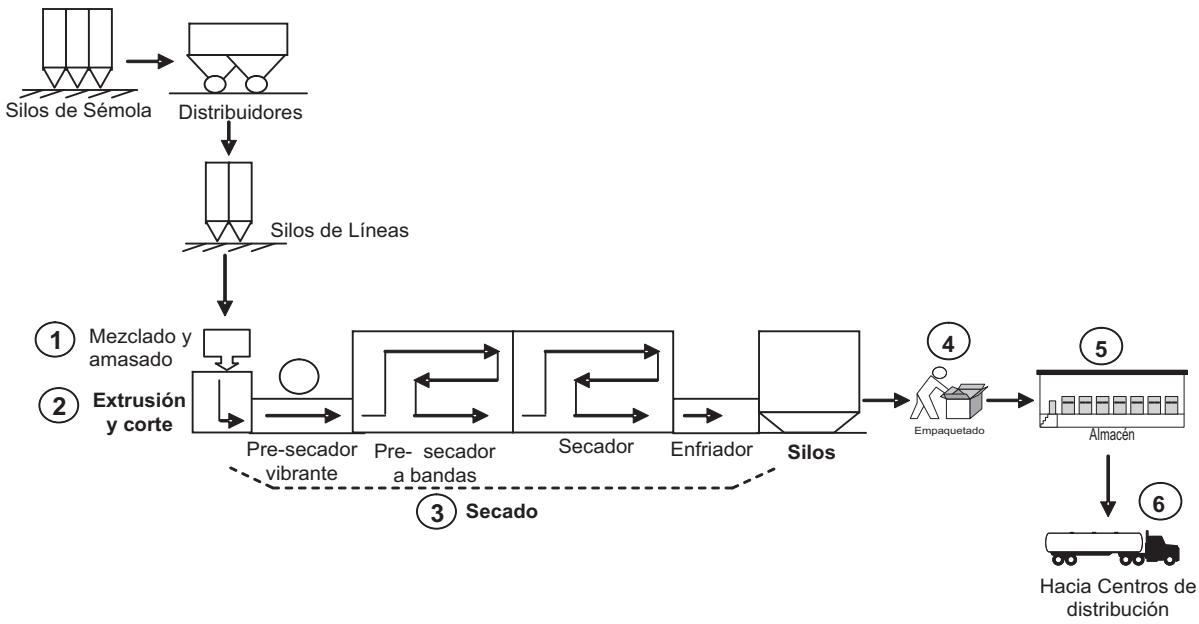

Fuente: Meleán (2004).

temente por adquirir tecnología de punta (maquinarias y equipos altamente especializados); la adopción de esta estrategia de modernización constante en las instalaciones productivas de las grandes empresas, se inició alrededor de los años
90, permitiendo la actualización de actividades productivas, el incremento de la productividad de sus procesos de trabajo y de los factores productivos, y la creación de ventajas competitivas, que según Krajewski y Ritzman (2000) cuando están 
representadas por herramientas tecnológicas pueden dar a la empresa una competencia difícil de igualar.

En estas industrias, las tecnologías adoptadas, están representadas por líneas de producción completamente automatizadas donde los equipos o departamentos se arreglan de acuerdo con los pasos progresivos mediante los cuales se hace el producto (Chase y col., 2000; Noori y Radford, 1997; Davis y col., 2001).

Dichas líneas, son controladas por dispositivos electrónicos denominados: Controladores Lógicos Programables (o PLC, por sus siglas en inglés); estos dispositivos, proporcionan alta confiabilidad, gran eficiencia y flexibilidad, además de presentar un mantenimiento económico. Los PLC, son definidos como una máquina electrónica diseñada para controlar en tiempo real y en medios industriales los procesos productivos; se emplean en situaciones complejas, de producción variable o secuencial, o que requieran de chequeos de programación centralizados en ciertas partes del proceso. Una de las ventajas más importantes de este tipo de dispositivos para los empresarios, es el reducido costo de mano de obra empleada en los procesos que se controlan (Vallejo, 2006).

Para Sorge (1993) y Fernández y col. (2006) la aplicación de modernas tecnologías en los proceso productivos de las empresas conlleva a la automatización de las instalaciones, facilita la productividad de las operaciones, la optimización continua del proceso y la gestión integral de la fábrica y sus sistemas adyacentes, integrando las funciones organizativas relacionadas con la fabricación. Además, los autores establecen que una de las principales razones por las cuales las empresas adoptan este tipo de tecnología es la gran flexibilidad que pueden lograr sin incurrir en elevados costos de fabricación.

La flexibilidad de los procesos de producción, puede interpretarse de diferentes formas. Si se consideran las industrias estudiadas, a pesar de que sus procesos están muy automatizados, la flexibilidad se presenta en escasa fases del proceso de fabricación: 1) el proceso está dispuesto en líneas de producción que solamente admiten cambios en la fase de extrusión y corte de la pasta, específicamente en el cambio de moldes ${ }^{7}$ que permiten obtener el formato ${ }^{8}$ de pasta que se desee; esto se conoce como: 1) flexibilidad de cambio y modificación, fabricando en la línea de pasta corta formatos como tornillo, pluma, dedal, caracol, codo entre otros y en la de pasta larga espagueti, vermicelli o fidellini, 2) existe una flexibilidad de materiales y de mezcla que permite alternar sémola o harina dependiendo de la calidad de la pasta a fabricar, y 3 ) se

7 Estos moldes permiten obtener un tipo de pasta con un formato específico; si es tornillo, plumitas, caracoles o cualquier otro tipo de pasta que se requiera en cada una de las líneas de producción para satisfacer las necesidades del mercado.

Formato, se refiere a la forma o gráfico de la pasta. 
presencia una flexibilidad en cuanto al volumen de producción de las líneas de fabricación tanto de pasta larga como de pasta corta dependiendo de las exigencias de los mercados (Fernández y col., 2006).

La estrategia de modernización de la producción adoptada por las grandes empresas, le ha permitido fundamentalmente: 1) mejorar el proceso de empacado; 2) mejorar diseños relativos al sellado del empaque; $y, 3$ ) incrementar la capacidad de producción acortando ciclos o tiempos en algunas fases de los procesos de trabajo que fomentan ahorros significativos en costos, principalmente de mano de obra no calificada; en tal sentido, Oz (2001) plantea que tal vez el logro más importante de las tecnologías de información en la producción es haber alcanzado una mayor agilidad del proceso productivo para satisfacer las necesidades del mercado.

Aunado a esto, las tecnologías de producción automatizadas han reemplazado parcialmente el trabajo que anteriormente hacían obreros calificados. Tal es el caso de una de las grandes empresas abordadas en el estudio, en la cual, la adquisición de este tipo tecnología le llevó a pasar de 12 a 4 trabajadores por turno. Hoy en día es suficiente un supervisor por línea de producción, los programadores u operarios de los equipos y el personal necesario para las funciones de mantenimiento; con esto se sustituye el trabajo humano poco calificado por líneas de producción completamente automatizadas. Esto se traduce en una reducción considerable de los costos de mano de obra asociados al proceso automatizado de elaboración de pastas alimenticias.
Sin embargo, al respecto, Coriat (1982) afirma que aunque la máquina posee la virtud económica de hacer el trabajo productivo, además de ser un instrumento de regularización y sometimiento de los trabajadores... no podrá suplirlo todo, aún el saber hacer es fundamental para muchas obras de la vida.

Para finalizar, Sorge (1993), plantea que el proceso de producción, constituye una rama de la tecnología que ha pasado de la innovación básica radical a la amplia difusión y madurez; debido básicamente a: 1) un empuje tecnológico que ha facilitado el uso de tecnologías duras (computadores, equipos de producción para controlar las funciones de manufactura, entre otros) que reemplazan esfuerzos físicos de personas no calificadas, por las denominadas tecnologías blandas representadas por el know-how o las habilidades y técnicas que se constituye en información que no necesariamente toma forma tangible (Gómez, 2006), reemplazando el esfuerzo físico por el conocimiento convertido en un arma competitiva en las empresas del siglo XXI; y 2) la presión de la demanda en la industria manufacturera, a utilizar máquinas y equipos de producción más productivos y al mismo tiempo más flexibles. A estos planteamientos, se puede agregar las exigencias de los mercados y la lucha de las empresas por crear ventajas competitivas sostenibles en el tiempo.

Otra forma de organizar el proceso productivo de elaboración de pasta larga y pasta corta, consiste en la aplicación de estrategias de reorganización. Estas estrategias son asumidas por las empresas cuando requieren modificar algunos de sus procesos. La reingeniería es una de 
ellas, considerada según Hammer y Champy (1994:2), como la "revisión fundamental y el rediseño radical de procesos para alcanzar mejoras espectaculares en medidas críticas y contemporáneas de rendimiento, tales como costos, calidad, servicio y rapidez". La reingeniería significa descartar todas las estructuras y los procedimientos existentes e inventar maneras enteramente nuevas de realizar un trabajo; reinventar el negocio, no modificarlo ni mejorarlo.

Sin embargo, las empresas analizadas, no son tan radicales ni arriesgadas para implantar cambios como los planteados por la reingeniería; asumen el rediseño o reorganización de partes, etapas o fases de los procesos, es decir, un rediseño parcial que se traduce en un mejoramiento continuo asumido con la finalidad de optimizar los procesos y hacerlos más eficientes, maximizar el desempeño, y lograr el éxito sostenido de cada una de las actividades que los integran.

Al respecto, los entrevistados comentan que "... en una situación en la cual se encuentran preocupados por sobrevivir y alcanzar una posición que les permita liderar mercados, no se atreven a implantar cambios radicales en los procesos (reingeniería)... más si, han realizado reorganizaciones importantes..." (Pernia, 2005).

Las estrategias de modernización productiva y las de reorganización, han permitido en las grandes empresas la actualización, rediseño y mejora en los procesos de trabajo que conforman el proceso de fabricación, elevando sus niveles de productividad y las posiciones competitivas en el mercado, producto de la creación de ventajas comparativas.
En la pequeña empresa, los paradigmas de organización familiar se encuentran muy arraigados por lo que debe comenzar por asumir una actitud de cambio ante las nuevas condiciones que el entorno empresarial impone, además de decidir sobre la aplicación de éste tipo de estrategias y de los beneficios implícitos a ella; pensar más en cambios representativos que en soluciones temporales a los problemas asociados a la no aplicación de las estrategias mencionadas.

A pesar de que la adopción de estrategias empresariales de modernización y reorganización, incrementan la productividad de los procesos productivos en las industrias analizadas, tienen consecuencias y/o efectos directos en el lado débil de la producción: la fuerza de trabajo, considerada factor esencial en todo proceso productivo. La mano de obra representa un factor cuya participación en el proceso de producción genera costos que dependen de la calificación, experticia y destreza que esta posea.

La automatización de las instalaciones desplaza mano de obra pero a la vez acelera el ritmo de las operaciones; esto incrementa los niveles de productividad y crea ventajas competitivas que no podría conseguir un obrero actuando por sí solo, sin el apoyo de las tecnologías de producción. En tal sentido, la tecnología constituye una herramienta que día tras día irá reemplazando el trabajo humano; lo que se traduce en un futuro incierto para el esfuerzo físico, un futuro donde sólo prevalecerá el conocimiento que tenga la mente humana, que se constituye en el principal activo creador de valor en cada una de las actividades que se realicen. La idea, es crear, como plantea 
Gamboa y col. (2003) una "tecnología con rostro humano" que no sólo aumente la productividad, sino que incite a las personas a ser más creativas y productivas en sí mismas...en última instancia una "tecnología que sirva al hombre en lugar de destruirlo".

Una vez comentada la estrategia de modernización productiva y algunas de reorganización se abordará la flexibilización de las relaciones laborales. Según estudios realizados, en Venezuela existe una tendencia a la flexibilización de la regulación del tiempo del trabajo; esto se verifica en la contratación colectiva y en propuestas legislativas (Iturraspe, 1991). Por otra parte, la reconversión industrial, así como la introducción de nuevas tecnologías traen como consecuencia mayores posibilidades de flexibilidad y un reforzamiento de las corrientes flexibilizadoras, para de esta manera configurar en Venezuela lo denominado por Sotelo (2003) el empleo justo a tiempo y la flexibilidad laboral.

La flexibilización laboral, de acuerdo con Battistini y Montes (2000), es una de las herramientas para adaptar las relaciones laborales al contexto productivo y especialmente a las condiciones del mercado; sin embargo, piensan que es una estrategia difícil de definir, debido a que se expresa de diferentes formas y depende de las circunstancias o contexto en que se trabaje.

Los autores citados tienen razón al decir que la flexibilización laboral va a depender de las circunstancias o contexto en que se trabaje. En Venezuela, la legislación laboral, no está creada para promover formas de flexibilización de las relaciones laborales como herramienta que desmejora la calidad de vida de los trabajadores; sin embargo, las empresas en una constante búsqueda por reducir sus costos, se apegan a ciertos artículos de la legislación laboral venezolana que permiten la contratación de personal por períodos determinados, para así reducir sus costos laborales, llegando a contratar personal en situaciones precarias de trabajo: contratos temporales, bajos salarios, ausencia de seguridad social, reducción del gasto en seguridad industrial, entre otros.

La flexibilización laboral es definida por Sotelo (1999:118), como un medio para hacer más competitivas a las empresas con cargo a las normativas de inseguridad en el empleo, el aumento en la intensidad del trabajo, la disminución de los salarios y la desregulación de las condiciones de protección de los trabajadores; esta última forma se asocia a la "...desigualdad del ingreso, pérdida de puestos de trabajo y diversas maneras en que las condiciones de los trabajadores se volvieron más precarias" y se va agudizando cuando no existen contrapesos políticos por parte de los sindicatos para frenar estas tendencias de reestructuración.

Según información suministrada por los gerentes de operaciones, conjuntamente con el gerente de recursos humanos, se evidencia que la estabilidad del personal en las empresas estudiadas es alta; el $100 \%$ del personal se considera contratado por periodos indefinidos en una de las empresas, en otra el $95 \%$ del personal es fijo y un $5 \%$ contratado; mientras que la tercera empresa no especificó la relación entre el personal contratado por tiempo determinado y el contratado por tiempo indeterminado, sin embargo 
explicó que ofrece los beneficios derivados de la contratación colectiva y discute contratos cada tres años de acuerdo con los cuales varian dichos beneficios (Pernía, 2005; Villalobos, 2005 y D’Falco, 2005).

Vale la pena destacar, de acuerdo con D'Falco (2005), que en la empresa pequeña la relación del personal fijo y contratado desde el año 2001 ha ido decreciendo, siendo ésta una de las empresas más afectada por el paro empresarial y petrolero del mismo año y por el golpe del año 2002. Por ello adopta una estrategia de outsourcing o subcontratación cuando requieren personal extra.

En una de las grandes empresas el número de trabajadores de planta se redujo en un $10 \%$ aproximadamente producto de la adquisición de nuevas tecnologías; mientras que en la empresa restante, el comportamiento relativo a la mano de obra (trabajadores de planta) fue similar, sin llegar a determinar el porcentaje de trabajadores desplazados por la adquisición de tecnología (Villalobos, 2005).

Aunque muchas veces, la adopción de ciertas estrategias conlleva al desplazamiento de la fuerza laboral, las empresas estudiadas afirman que en lo posible tratan de no afectar las condiciones ni los beneficios de la fuerza de trabajo.

La flexibilización de las relaciones laborales, atenta contra los empleos duraderos y contra la calidad de vida de los trabajadores menos preparados, y cada vez su aplicación es mayor en el mercado laboral. Sin embargo, en las empresas analizadas no se pudo determinar con exactitud la existencia de estrategias que flexibilizan las relaciones laborales, dada la confidencialidad que mantienen las grandes empresas con este tipo de información. Sin embargo, la proporción de trabajadores fijos sugiere poca utilización de esta estrategia, por lo cual se puede afirmar según la información suministrada que estas empresas prevalece la estabilidad laboral.

\subsection{Producción artesanal de especialidades}

Desde sus inicios, la producción estaba circunscrita a la artesanía o producción artesanal, basada en la idea de que las máquinas y los procesos podian aumentar las calificaciones del artesano y permitir así plasmar sus conocimientos en productos cada vez más diversos: cuanto más flexible era la máquina y más amplias las posibilidades de aplicación del proceso, más aumentaba la capacidad del artesano para la expresión productiva (Piore y Sabel, 1990, citado por Chacón y col., 2006).

Con el transcurrir de los años, se inicia la producción en serie que adopta herramientas de trabajo operados manualmente por el hombre pero que utiliza dispositivos mecánicos; se comienzan a dividir las tareas en el área productiva y a reemplazarse un saber de fabricación o know how por tareas específicas y repetitivas que incrementarían la productividad del proceso productivo en la empresa, y en consecuencia la rentabilidad del gran capitalista. En tal sentido, se pierde el dominio de un oficio y se comienzan a producir partes o componentes específicas de un determinado producto, existe la estandarización y penalizaciones por los desperdicios, principalmente en tiempos, durante el proceso de producción. Según 
Coriat (1982), doblegar el obrero de oficio, "liberar" al proceso de trabajo del poder que éste ejerce sobre él para instalar en su lugar la ley y la norma patronal, será la contribución histórica del taylorismo.

A pesar del desplazamiento que han tenido los procesos de producción artesanales en diferentes organizaciones, debido a la adopción de maquinarias y equipos que incrementan ritmos de trabajo, reducen ineficiencias y aumentan los niveles de productividad de los procesos, aspectos que se traducen en una reducción significativa de costos de producción, (específicamente laborales); existen aún empresas que requieren de éste tipo de proceso para obtener productos con características específicas que sería imposible (hasta ahora) conseguir con tecnología automatizada. Como plantea Novick (2005), la gran empresa con su homogeneidad tecnológica y productiva se constituye en eje central; pero las empresas de carácter artesanal, constituyen el complemento obligado de la gran empresa.

El proceso de producción artesanal, a diferencia de la producción automatizada, requiere de fuerza de trabajo cualificada y polivalente en grandes cantidades. Esta mano de obra domina un oficio (por lo general adquirido en familia), característica imprescindible para llevar a cabo este tipo de proceso; el conocimiento es esencial para obtener un control total de la actividad productiva y no de una parte específica de la misma. La fortaleza representada por el dominio del saber no es expropiable y permite controlar el tiem- po de trabajo (Lucena, 2003 y Fernández y col., 2003). En lo concerniente a los medios de producción utilizados, están representados por herramientas simples y rudimentarias que apoyan las actividades necesarias para lograr un producto con determinadas características.

Según Fernández y col. (2006), en el artesanado, la producción se caracteriza por un volumen relativamente bajo de producción ${ }^{9}$ (producción a pequeña escala) y la amplia variedad de diseños destinados a satisfacer nichos de mercados específicos.

Hay que tomar en cuenta, que la evolución de los procesos productivos ha logrado agregar a los sistemas artesanales características propias de otros momentos históricos; la organización de la producción, la reducción de los desperdicios, la acumulación de capital y el incremento de los niveles productividad, factores sumamente importantes para lograr metas de rendimiento financiero y garantizar la permanencia de las empresas en el mercado.

Los procesos de producción artesanales, dado el esfuerzo y el conocimiento requerido para llevarlos a cabo, en grandes ocasiones son externalizados o delegados en terceras personas, aprovechando los beneficios derivados de la aplicación de esta estrategia empresarial; más aún, si el resto de los procesos productivos de las empresas que requieren de los productos derivados de dicho proceso son completamente automatizados y/o mecanizados. 
La externalización, también denominada subcontratación u outsourcing según Fernández y col (2006:334) consiste en "dejar en manos de profesionales externos, generalmente especialistas, la realización de determinadas funciones que no son clave para la empresa"; la finalidad de esta estrategia radica en la optimización de recursos actuales y la reducción de costos. Al respecto Chase y col. (2005) agrega que con las transferencias de actividades internas, se transfieren igualmente responsabilidades de decisión de la compañía a otras empresas, en este caso proveedores externos, estableciendo los términos del acuerdo en un contrato.

Las principales razones para externalizar actividades o proceso no medulares, se traducen fundamentalmente en costos, sin perder de vista las asociadas a la creación de ventajas competitivas que se logran con la concentración en actividades esenciales, sobre las cuales la empresa se especializa.

En el sector industrial analizado, las grandes empresas subcontratan agrupaciones de carácter artesanal para la fabricación de las especialidades que ampliarán la cartera de productos ofrecidos con sus marcas en el mercado. En tal sentido, se desligan de esta actividad transfiriéndola a especialistas con ventajas competitivas reconocibles en el desarrollo de esta actividad. Con la externalización, las empresas que subcontratan se evitan realizar inversiones en activos fijos cuya relación costo beneficio tal vez no sea la más adecuada, dadas las particularidades del proceso subcontratado.

Las peculiaridades de las especialidades, entre ellas: los ñoquis de papa, raviolis, tortelloni, pasta al huevo, entre otros, requieren de un proceso artesanal que no puede ser realizado por las sofisticadas líneas de producción que poseen dichas empresas; sin embargo, dichos productos son necesarios para atender el segmento del mercado que los consume y ampliar la diversidad de productos del ramo alimenticio ofrecidos por estas empresas.

El proceso productivo para la elaboración de éstas especialidades requiere de grandes destrezas y/o habilidades manuales ofrecidas por artesanos (representado en este caso por mujeres) dada la forma exacta que requiere la masa para obtener productos con determinadas características y aceptación por parte de los consumidores.

Otro aspecto que forma parte del proceso de elaboración de las especialidades son los ingredientes (adicionales a la mezcla de harina o sémola) para el relleno de algunos productos; son amplios los diseños y productos al igual que el trabajo requerido para obtenerlos.

La idea de las empresas es ofrecer productos en el mercado con sus marcas, para ampliar la variedad que conforma su cartera, más que por las ganancias netas derivadas por la venta de dichas especialidades; sin embargo, las razones fundamentales para subcontratar el proceso artesanal de fabricación de especialidades radican básicamente en: 1) la reducción de costos a través de un desempeño superior del proveedor; 2) la reducción de inversiones en activos y utilización de dichos recursos en otros proyectos; y 3) concentración de energías en los procesos medulares de la organización. 


\section{Conclusiones}

Una vez finalizada la investigación se pueden establecer las siguientes conclusiones:

- El sector industrial de pastas alimenticias está representado por tres empresas, dos de las cuales son consideradas grandes de acuerdo con el artículo 3 de la Ley para la promoción y desarrollo de la pequeña y mediana industria mientas que la tercera es una empresa de carácter familiar catalogada como pequeña. Dichas empresas fabrican pastas alimenticias en diferentes formatos y presentaciones destinados a satisfacer necesidades alimenticias de la población.

- En las empresas analizadas, los procesos de producción se dividen en: el proceso de elaboración de pasta larga y el de pasta corta en sus diferentes formatos y presentaciones; y el proceso de elaboración de las especialidades.

- Los procesos productivos para la fabricación de la pasta larga y la pasta corta son similares en las empresas analizadas. La elaboración de pasta en las grandes empresas se desarrolla en líneas de producción completamente automatizadas, controladas por dispositivos electrónicos que controlan armónicamente la producción (PLC), mientras que en la empresa pequeña, la tecnología que apoya los procesos productivos es mecaniza$\mathrm{da}$, y presenta gran retraso comparada con la del sector.

- En lo que respecta a la elaboración de especialidades, las grandes empresas con la finalidad de ampliar la di- versidad de productos ofrecidos en el mercado subcontrata la producción de las mismas externalizando dicho proceso en empresas artesanales.

- Las empresas en una constante presión por organizar adecuadamente sus procesos productivos, se han valido de estrategias empresariales, básicamente de modernización tecnológica en producción y de reorganización de procesos empresariales. Estas han sido aplicadas con éxito en las grandes empresas, mejorando procesos como el empacado y sellado de los empaques, entre otros; esto, incrementa la capacidad de producción, acortando ciclos o tiempos en algunas fases de los mismos.

- Las estrategias de modernización tecnológica en producción, conjuntamente con la de reorganización fomentan ahorros significativos en costos, principalmente de mano de obra no calificada, siendo éstos uno de los beneficios más importantes para los empresarios. La adquisición de tecnología permite a las empresas: alcanzar una mayor agilidad del proceso productivo para satisfacer las necesidades del mercado y reducir de costos asociados a la mano de obra partícipe en los procesos.

- La empresa pequeña no ha realizado inversiones significativas en tecnología de producción, simplemente se limita a realizar adaptaciones (por los mismos trabajadores) a la maquinaria para mantenerla operativa.

- Las estrategias de modernización y reorganización, sin duda orientan a las empresas que las adoptan (grandes empresas) a reemplazar la fuerza 
de trabajo por tecnología automatizada; esto reduce costos de producción, asociados a la mano de obra; sin embargo, el personal desplazado no es suficiente para hablar de una flexibilización numérica, predominando en estas empresas la estabilidad laboral.

- La estrategia de externalización asumida por las empresas para la elaboración de las especialidades, permite la creación de ventajas competitivas asociadas a la concentración de esfuerzos en las actividades consideradas esenciales. Al subcontratar procesos no medulares las empresas delegan en proveedores especializados y con ventajas competitivas comprobables dichos procesos, cuya ejecución se puede lograr con mayores ventajas y con costos reducidos.

\section{Referencias Bibliográficas}

Asamblea Nacional de la República Bolivariana de Venezuela (2006). Ley para la promoción y desarrollo de la pequeña y mediana industria. Fuente: http://www.asambleanacional.gov.ve Ins2/leyes. $a s p ? i d=474$ Fecha de consulta: 15/01/06.

Asociación Venezolana de Fabricantes de Pastas (2001). Pasta en Venezuela. http://www.avepastas.org.ve/Pastas/ pasta.html Fecha de consulta: 15/05/ 2001.

Battistini, Osvaldo y Montes Cato, Juan (2000). Flexibilización Laboral en Argentina. Un camino hacia la precarización y desocupación. Revista Venezolana de Gerencia. Año 5. № 10. Páginas 63-89. Universidad del Zulia. Maracaibo, Venezuela.
Chacón, Galia; Bustos, Carlos y Rojas, Elí S. (2006). Los proceso de producción y la contabilidad de costos. Revista Actualidad contable. FACES Año 9 № 12. Enero-Junio 2006. Mérida-Venezuela (16-26).

Chase, Richard; Aquilano, Nicolas y Jacobs, Robert (2000). Administración de Producción y Operaciones. McGraw-Hill Interamericana, S.A. Octava Edición. Colombia. Pág. 885.

Chase, Richard; Aquilano, Nicolas y Jacobs, Robert (2005). Administración de la Producción y Operaciones. Para una ventaja competitiva McGraw-Hill Interamericana, S.A. Décima Edición. Colombia. Pág. 885.

Coriat, Benjamin (1982). El Taller y el cronómetro. Ensayo sobre el Taylorismo, el fordismo y la producción en masa. Siglo Veintiuno Editores. 2da Edición. México.

Davis, Marck M.; Aquilano, Nicholas J. y Chase, Richard B. (2001). Fundamentos de Dirección de Operaciones. Tercera Edición. McGraw-Hill. España.

Diccionario de la Real Academia Española (2006). Vigésima Segunda Edición Fuente: http://www.rae.es/ Fecha de consulta: 05/0706

Diccionario el mundo (2006) Fuente: http://diccionarios.elmundo.es/ Fecha de consulta: 05/0706

Fernández, Esteban; Avella, Lucia y Fernández, Marta (2003). Estrategia de Producción. McGraw-Hill. Madrid, España.

Fernández, Esteban; Avella, Lucía y Fernández, Marta (2006) Estrategia de Producción. Segunda Edición McGrawHill. Madrid, España.

Gamboa Cáceres, Teresa; Arellano Rodríguez, Madelein y Nava Vásquez, Yuneska (2001). Estrategias Empresariales: Aproximación a una Tipología. Revista Venezolana de Ciencias 
Sociales. Volumen 5 No. 2. Universidad Nacional Experimental Rafael María Baralt. Páginas 132-154. Maracaibo, Venezuela.

Gamboa Cáceres, Teresa; Arellano Rodríguez, Madelein y Nava Vásquez, Yuneska (2003). Estrategias de modernización empresarial: Procesos, productos y fuerza de trabajo. Revista Venezolana de Gerencia. Año 8 No. 24. Universidad del Zulia. Páginas 592-606. Maracaibo, Venezuela.

Gamboa Cáceres, Teresa; Arellano Rodríguez, Madelein y Nava Vásquez, Yuneska (2003). Actores y fines de las estrategias empresariales. Una reflexión desde las pequeñas y medianas empresas. Revista Visión Gerencial del Centro de Investigaciones y Desarrollo Empresarial (CIDE). Año 2 No. 1. Vol. 1 Enero-Junio. Universidad de los Andes. Páginas 2839. Mérida-Venezuela.

Gómez, María (2006). Uso de las tecnologías duras y blandas en la organización. Fuente: http://servicios.universia.edu. ve/foros/viewtopic.php?t=1995\&start $=15 \&$, Fecha de consulta: 21/07/06.

Hammer, Michael y Champy, James (1994). Reingeniería. Olvide lo que usted sabe sobre cómo debe funcionar una empresa. ¡Casi todo está errado! Grupo Editorial Norma. Bogotá, Colombia.

Iturraspe, Francisco José (1991). "Relaciones Laborales en el mercado de trabajo urbano: inserción laboral en barrios segregados de Venezuela". En: Galin, Montero, Villasmil, Iturraspe, Freitez, lacabana, Montoliú, alonso (1991) Flexibilización Laboral en Venezuela. ¿Nuevo nombre o nueva realidad? Editorial Nueva Sociedad (Instituto Latinoamericano de Investigaciones Sociales (ILDIS). Primera Edición.
Krajewski, Lee J. y Ritzman, Larry P. (2000). Administración de Operaciones. Estrategia y Análisis. Quinta Edición. México.

Lucena, Héctor (1999). Modernización Productiva y Negociaciones Colectivas. Ediciones Universidad de Carabobo. Instituto Latinoamericano de Investigaciones Sociales (ILDIS) Valencia-Venezuela.

Lucena, Héctor (2003). Relaciones de Trabajo en el nuevo siglo. Fondo Editorial Tropykos. Caracas-Venezuela.

Meleán Romero, Rosana A. (2004). Estrategias para gerenciar costos de producción en industrias de pastas alimenticias. Trabajo Especial de Grado. División de Estudios para Graduados. Facultad de Ciencias Económicas y Sociales. Universidad del Zulia. Maracaibo-Venezuela.

Noori, Hamid y Radford, Russell (1997). Administración de Operaciones y Producción: Calidad Total y respuesta sensible rápida. Editorial McGrawHill. Santafé de Bogotá, Colombia.

Novick, Martha (2005). Una mirada integradora de las relaciones entre empresas y competencias laborales en América latina. Consejo Nacional de investigaciones científicas y técnicas (CONICET) Facultad de Ciencias Sociales. Universidad de Buenos Aires. Argentina. Fuente: http://www.cinterfor.org.uy/public/ spanish/region/ampro/cinterfor/publ/novick/pdf/novnovic.pdf Consulta: 27/04/05

Oz, Effy (2001). Administración de Sistemas de Información Segunda Edición. Editorial Thompson Learning. México.

Rodríguez, Guillermo; Balestrini, Solange; Balestrini, Sara; Meleán, Rosana y Rodríguez, Belkis (2002). Análisis Estratégico del Proceso Productivo en el Sector Industrial. Revista de Ciencias Sociales. Volumen VIII. 
No. 1. Enero-Abril. Páginas 135-156. Universidad del Zulia.

Sipper, Daniel y Bulfin Jr. Robert L. (1998). Planeación y Control de la Producción. Editorial McGraw-Hill. Mexico. Páginas 657.

Sorge, Arndt (1993). "La tecnología de producción en Europa: contexto social e industrial". En: Dombois, Rainer y Pries, Ludger. Modernización Empresarial: tendencias en América Latina y Europa. Fundación Frieddrich Ebert de Colombia (FESCOL). Editorial Nueva Sociedad. Colombia.

Sotelo Valencia, Adrián (1999). Globalización y Precariedad del Trabajo en México. Ediciones el Caballito. México.

Sotelo Valencia, Adrián (2003). La Reestructuración del Mundo del Trabajo. Superexplotación y nuevos paradigmas de la organización del trabajo. Editorial Itaca. México.

Tamayo Arocha, Francisco (1991). Gestión de manufactura. Nuevos enfoques de racionalización. Corporación Andina de Fomento Editorial Nuevos tiempos. Venezuela.

Vallejo, Horacio (2006). Los controladores lógicos programables. Fuente: http://www.todopic.com.ar/utiles/

PLC.pdf. Fecha de consulta: 06/07/06

Yip, George S. (1993). Globalización. Una estrategia para obtener una ventaja competitiva internacional. Editorial Norma. Bogotá-Colombia. Pág.331.

\section{Entrevistas realizadas:}

Pernía, Jhonny (2005). Gerente de calidad de la empresa Cargill de Venezuela. Fecha de la entrevista: 25/11/0527/04/06.

Villalobos, Jorge (2005). Gerente de planta de la empresa Molinos Sagra, C.A. Fecha de la entrevista: 23/06/04.

D'Falco, Erick (2005). Gerente general de la empresa La Comisana Fecha de la entrevista: 21/10/05. 